\title{
Synthesis of 2,3-di-O-( $\beta$-D-Galp)-D-Galp, a synthon for the mucin oligosaccharides of Trypanosoma cruzi
}

\author{
Verónica M. Mendoza, Carola Gallo-Rodriguez, and Rosa M. de Lederkremer* \\ CIHIDECAR. Departamento de Química Orgánica, Facultad de Ciencias Exactas y Naturales, \\ Universidad de Buenos Aires, Ciudad Universitaria, Pabellón II, 1428 Buenos Aires, Argentina \\ E-mail: lederk@qo.fcen.uba.ar
}

\section{Dedicated to Prof. Roberto Rossi on his $60^{\text {th }}$ Anniversary and Prof. Edmundo Rúveda on his $70^{\text {th }}$ Anniversary \\ (received 10 Jun 03; accepted 22 Jul 03; published on the web 25 Jul 03)}

\begin{abstract}
The synthesis of an anomeric free derivative of 2,3-di-O-( $\beta$-D-Galp)-D-Galp as a synthon for the mucin oligosaccharides of Trypanosoma cruzi is described. The title compound was synthesized via two approaches. The first one involved 2,3-di-O-glycosylation of 5,6-O-isopropylidene-Dgalactono-1,4-lactone by $O$-(2,3,4,6-tetra- $O$-acetyl- $\alpha$-D-galactopyranosyl) trichloroacetimidate. The second approach was based on glycosylation of a convenient derivative of galactopyranose. The synthesized trisaccharide is the acceptor unit of sialic acid in the trans-sialidase reaction that takes place in T. cruzi.
\end{abstract}

Keywords: Galactose trisaccharide, mucins, Trypanosoma cruzi, D-galactono-1,4-lactone, glycosylation, trichloroacetimidate

\section{Introduction}

The mucin-like glycoproteins are major components in the surface of Trypanosoma cruzi, the agent of American trypanosomiasis. ${ }^{1}$ The oligosaccharides in the mucins are $O$-linked to the protein via GlcNAc which is further substituted with galactose. Most of the Gal residues are present as $\beta$-Galp although in some strains a $\beta$-Gal $f$ residue is also present. ${ }^{2,3}$ The terminal $\beta$ Galp residues are acceptors of sialic acid in the trans-sialidase reaction, involved in the invasion of the host cells. ${ }^{4,5}$ In the past few years we have been working on the synthesis of galactofuranose-containing oligosaccharides, components of the mucins of the G-strain. ${ }^{6-8}$ From the same strain a penta- and a hexasaccharide (Figure 1) have been obtained as alditols by reductive $\beta$-elimination of the mucins. ${ }^{3}$ The trisaccharide 2,3 -di- $O$ - $(\beta$-D-galactopyranosyl)-Dgalactose is a common unit in the larger oligosaccharides, and a site for sialylation. It has been 
also recently characterized in the Dm28C strain. ${ }^{9}$ The same trisaccharide, present as a triterpene glycoside in a sponge of the genus Erylus, has been characterized by two dimensional NMR spectroscopy. ${ }^{10}$ A 3-(methoxycarbonyl)propyl $\alpha$-glycoside of the trisaccharide has been obtained as a by-product in a photochemistry study. ${ }^{11}$
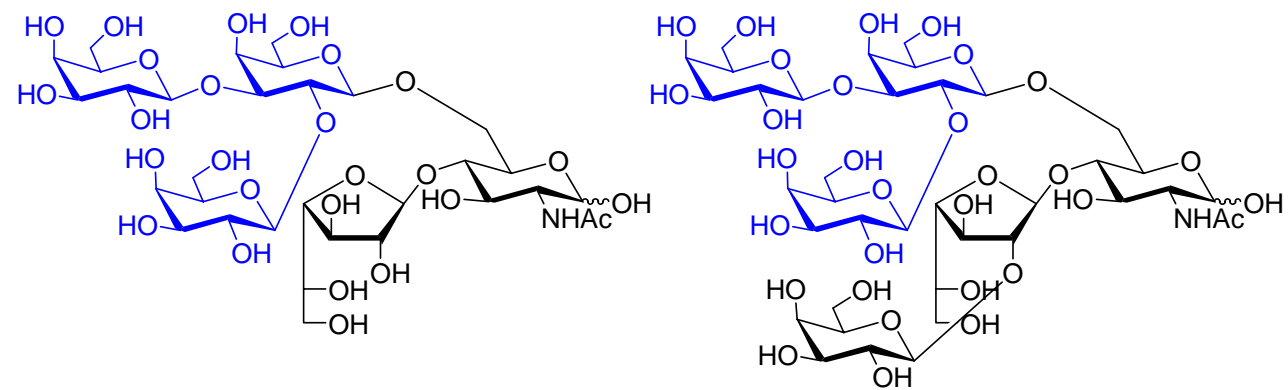

Figure 1. Structure of the penta and hexasaccharide in mucins of T. cruzi (G strain). The trisaccharide synthon is shown in blue.

In this paper we describe the synthesis of 4,6-di-O-acetyl-2,3-di-O-(2,3,4,6-tetra- $O$-acetyl- $\beta$ D-galactopyranosyl)-D-galactopyranose (8) as a synthon for the construction of the penta and hexasaccharide of the mucins of the $\mathrm{G}^{2,3}$ and $\mathrm{Dm} 28 \mathrm{C}^{9}$ strains. The free trisaccharide 14 obtained in this study, will be evaluated as sialic acid acceptor.

\section{Results and Discussion}

For the synthesis of trisaccharide 8, two approaches were evaluated. The first attempt involved the aldonolactone approach. Aldonolactones are selectively substituted and are good precursors for the reducing end. Recently, we synthesized a $\beta$-D-Galp-(1-3)-D-Galp fragment using Dgalactono-1,4-lactone as precursor for the Galp unit, taking advantage of the facility to obtain a 3-OH free crystalline derivative from this lactone. ${ }^{8}$ In the present case, for the synthesis of the target trisaccharide 8, 5,6-O-isopropylidene-D-galactono-1,4-lactone $(\mathbf{1})^{12}$ was employed as acceptor, and the trichloroacetimidate method of glycosylation ${ }^{13}$ was used (Scheme 1). Thus, glycosylation with 2.4 equivalents of $O$-(2,3,4,6-tetra- $O$-acetyl- $\alpha$-D-galactopyranosyl) trichloroacetimidate (2) ${ }^{14}$ with a catalytic amount of TMSOTf gave 2,3-di-O-(2,3,4,6-tetra-Oacetyl- $\beta$-D-galactopyranosyl)-5,6-O-isopropylidene-D-galactono-1,4-lactone (3) with $82 \%$ yield. The ${ }^{13} \mathrm{C}$ NMR spectrum showed the two resonances of the anomeric carbons at 101.0 and 99.4 ppm. The $\beta$-pyranosic configurations were confirmed by the ${ }^{1} \mathrm{H}$ NMR spectrum as indicated by the two doublets $(J=7.9$ and $7.8 \mathrm{~Hz})$ centered at 4.73 and $4.72 \mathrm{ppm}$ for $\mathrm{H}-1^{\prime}$ and $\mathrm{H}-1^{\prime \prime}$.

With the lactonic trisaccharide $\mathbf{3}$ in hand, the next steps were to reduce the lactonic function and to isomerize the furanosic reducing end to the pyranosic configuration. Thus, reduction of 3 with diisoamylborane ${ }^{15}$ afforded the trisaccharide derivative $\mathbf{4}$ purified by column chromatography in $57 \%$ yield, together with 2,3-di-O-(2,3,4,6-tetra-O-acetyl- $\alpha$-D- 
galactopyranosyl)-D-galactose $(6,14 \%)$, and its precursor 2,3-di-O-(2,3,4,6-tetra- $O$-acetyl- $\beta$-Dgalactopyranosyl)-D-galactono-1,4-lactone (5, $15 \%)$. The steric hindrance due to substitution in positions-2,3 of the furanosic ring in compounds $\mathbf{3}$ and $\mathbf{4}$ must be responsible for the lability of the isopropylidene group giving 5 and $\mathbf{6}$. In previous work from our laboratory isopropylidene lactone derivatives showed to be stable. , $16^{-16}$

Compound 4 was hydrolyzed to 6 by heating at $60{ }^{\circ} \mathrm{C}$ with aqueous acetic acid. The ${ }^{13} \mathrm{C}$ NMR spectrum of 6 showed the resonances for the reducing end anomeric carbons at $95.5(\mathrm{C}-1 \alpha$ furanosic), 94.8 (C-1 $\beta$ pyranosic), $92.5 \mathrm{ppm}$ (C-1 $\alpha$ pyranosic). The signal for $\mathrm{C}-1 \beta$ furanosic was overlapped at $\sim 100 \mathrm{ppm}$ with the signals for the anomeric carbons of the $\beta$-Galp substituents. The anomeric composition could not be estimated from the ${ }^{1} \mathrm{H}$ NMR spectrum because of the high superposition of signals.

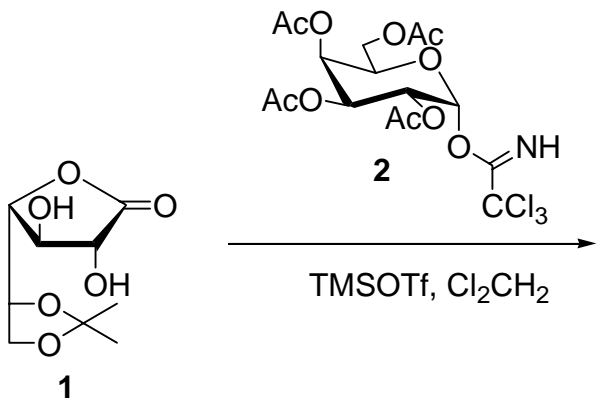

$\underset{60{ }^{\circ} \mathrm{C}}{\stackrel{\mathrm{AcOH} / \mathrm{H}_{2}}{\longrightarrow}}$

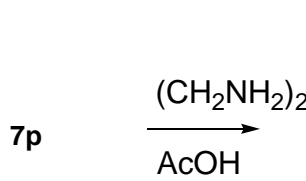

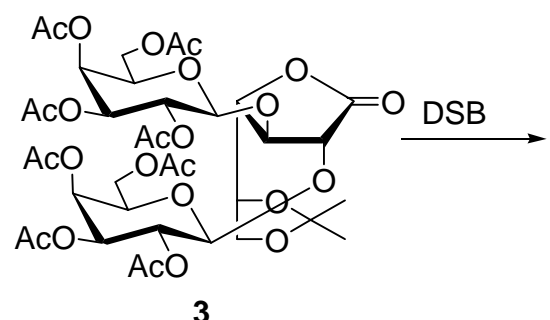

3

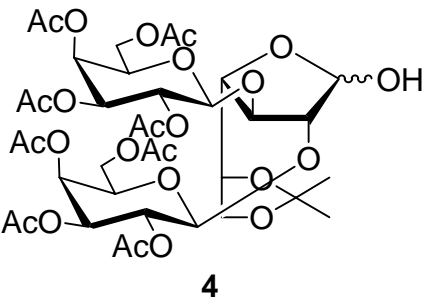

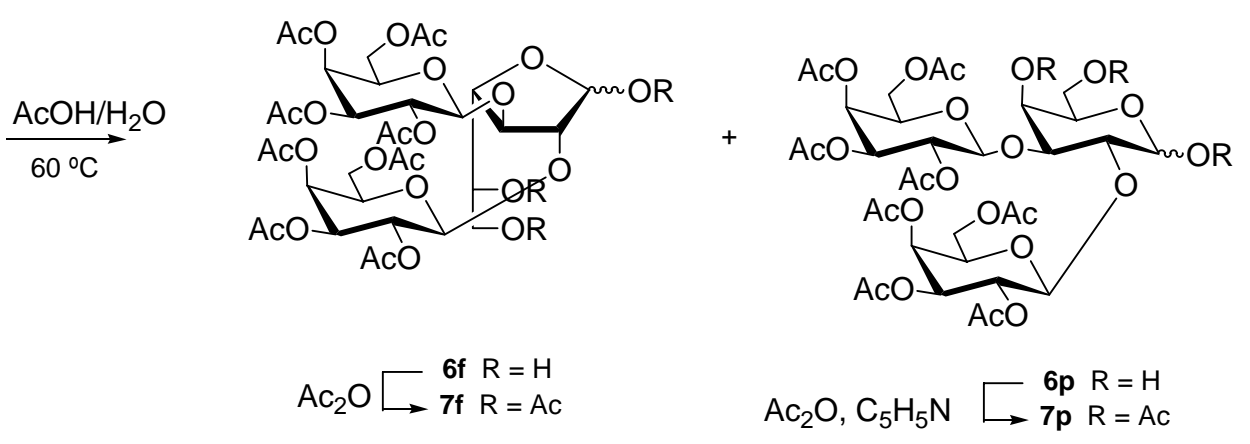

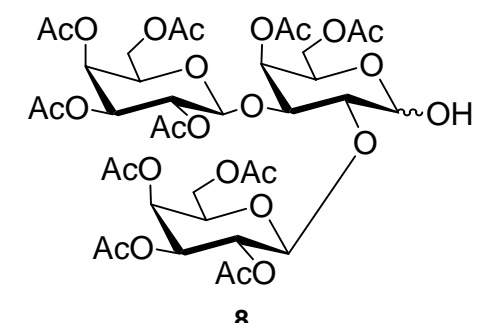

8

Scheme 1. Synthesis of 8 by the aldonolactone approach. 
In order to obtain mainly the pyranosic configuration at the reducing end, acetylation of 6 was performed in pyridine at low temperature. The crude product was a mixture of $\beta$ furanosic: $\alpha$-furanosic: $\beta$-pyranosic: $\alpha$-pyranosic acetates in 34:20:6:40 ratio as shown by integration of the anomeric protons in the ${ }^{1} \mathrm{H}$ NMR spectrum: $\delta 6.36(\mathrm{~d}, J=3.8 \mathrm{~Hz}, \mathrm{H}-1 \alpha$ pyranosic), 6.31 (bs, H-1 $\beta$ furanosic), 6.17 (d, $J=4.2 \mathrm{~Hz}, \mathrm{H}-1 \alpha$ furanosic), 5.63 (d, J=7.6 Hz, $\mathrm{H}-1 \beta$ pyranosic). The poor isomerization to the pyranosic configuration could be related to the high steric hindrance caused by the 2,3-di-O-Galp substitutions. In fact, acetylation of an analogous derivative of $\beta$-D-Galp-(1-3)-D-Gal afforded the pyranosic acetate form in $80 \%$ yield. ${ }^{8}$

The last step for the synthesis of synthon $\mathbf{8}$ was the hydrolysis of the anomeric acetate which was accomplished by treatment of $\mathbf{7 p}$ with ethylenediamine-acetic acid (Kováč's procedure). ${ }^{17}$ Compound 8 was obtained in the $\alpha$-anomeric configuration as shown in the ${ }^{1} \mathrm{H}$ NMR spectrum.

The low proportion obtained (46\%) of the pyranosic trisaccharide $\mathbf{7 p}$ and its difficult purification led us to look for another route. As starting material benzyl 4,6-di-O-benzylidene- $\beta$ D-galactopyranoside ${ }^{18}(\mathbf{9})$, obtained in three steps from penta- $O$-acetyl- $\alpha, \beta$-D-galactose, was employed. The use of a benzyl glycoside provides the access to the anomeric free trisaccharide necessary for further condensation by the trichloroacetimidate method. Thus, glycosylation of 9 with 2.4 equivalents of trichloroacetimidate 2 gave trisaccharide benzyl 4,6-O-benzylidene-2,3di-O-(2,3,4,6-tetra- $O$-acetyl- $\beta$-D-galactopyranosyl)- $\beta$-D-galactopyranoside (10) in $59 \%$ yield (Scheme 2). In the ${ }^{13} \mathrm{C}$ NMR spectrum the three $\beta$-galactopyranosic anomeric carbons resonate at $100.7,100.3$, and $99.7 \mathrm{ppm}$. On the other hand, the ${ }^{1} \mathrm{H}$ NMR spectrum showed three doublets at 4.92, 4.85 and $4.56 \mathrm{ppm}$ with coupling constants of 7.7, 8.0 and $7.7 \mathrm{~Hz}$ respectively, that confirmed the $\beta$-pyranosic assignments. Glycosylation of compound 9 by the Köenigs-Knorr reaction was described. ${ }^{19}$ In that case, the two possible disaccharide derivatives were obtained, by $2-O$ or 3-O-mono-substitution with a Galp residue. Di-O-substitution that would afford compound 10 was not reported.

Further hydrolysis of the benzylidene of 10, followed by acetylation of the product gave benzyl 4,6-di-O-acetyl-2,3-di-O-(2,3,4,6-tetra- $O$-acetyl- $\beta$-D-galactopyranosyl)- $\beta$-D-galactopyranoside (12) in $68 \%$ yield after the two steps. Hydrogenolysis of the benzyl glycoside of 12 gave the anomeric free trisaccharide $\mathbf{8}$, in this case in the $\beta$-configuration. Isomerization of the anomeric carbon in a chloroformic solution for a week gave a 3:1 $\beta / \alpha$ mixture.

Finally, the free trisaccharide 2,3-di-O- $\beta$-D-galactopyranosyl- $\beta$-D-galactose (14) was synthesized (Scheme 3). Thus, treatment of 12 with sodium methoxide gave crystalline benzyl 2,3-di-O- $\beta$-D-galactopyranosyl- $\beta$-D-galactopyranoside (13) in $90 \%$ yield. Hydrogenolysis of 13 with $\mathrm{H}_{2} / \mathrm{Pd}(\mathrm{C})$ gave the crystalline trisaccharide 14 as a mixture of the four anomers $\beta$ furanosic: $\alpha$-furanosic: $\beta$-pyranosic: $\alpha$-pyranosic in a 12:5:25:58 ratio. 


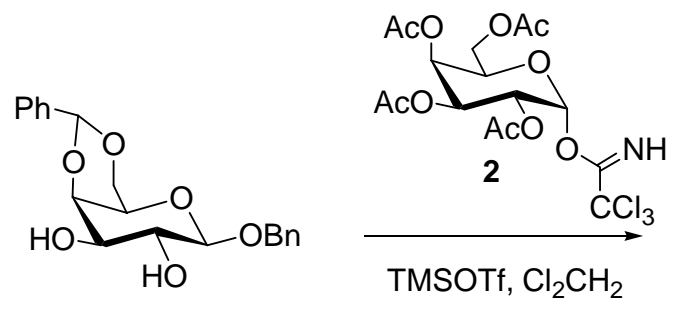

9

AcO OAC OR OR

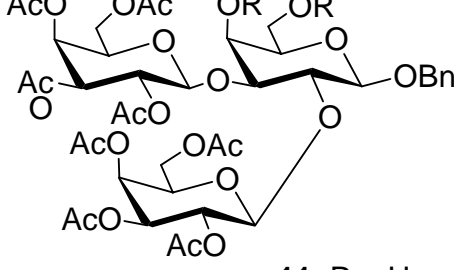

$\mathrm{Ac}_{2} \mathrm{O}, \mathrm{C}_{5} \mathrm{H}_{5} \mathrm{~N} \longrightarrow \begin{array}{ll}11 \mathrm{R}=\mathrm{H} \\ 12 \mathrm{R}=\mathrm{Ac}\end{array}$

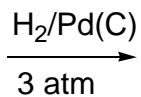

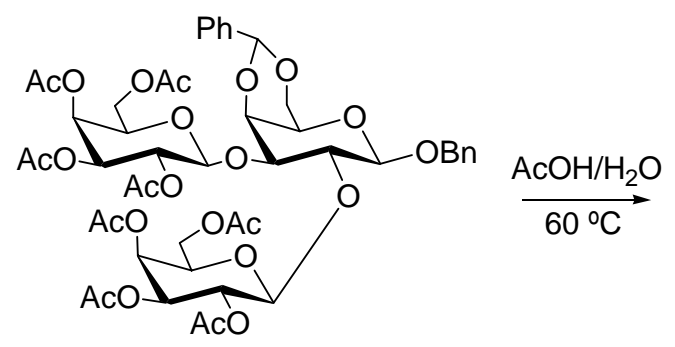

10

8

Scheme 2

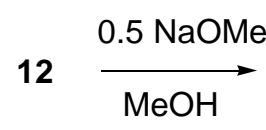

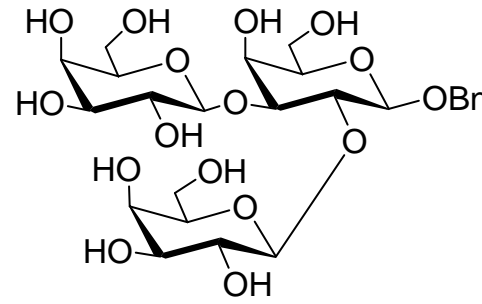

13

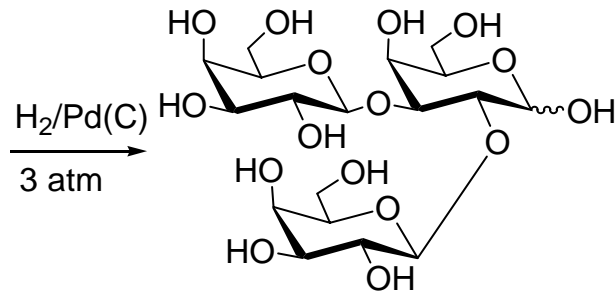

14

\section{Scheme 3}

In conclusion, in this work we synthesized the trisaccharide synthon, precursor of the penta and hexasaccharide constituents of the mucins from T. cruzi (Figure 1) via the glycosylaldonolactone approach that included a Galp isomerization and by a classical approach. The free trisaccharide 2,3-di-O- $\beta$-D-galactopyranosyl- $\beta$-D-galactopyranoside (14) was also synthesized as a useful tool for studying its acceptor properties in the trans-sialidase reaction.

\section{Experimental Section}

General Procedures. TLC was performed on $0.2 \mathrm{~mm}$ silica gel 60 F254 (Merck) aluminum supported plates. Detection was effected by exposure to UV light or by spraying with $10 \%$ (v/v) sulfuric acid in EtOH and charring. Column chromatography was performed on silica gel 60 (230-400 mesh, Merck). Melting points were determined with a Thomas-Hoover apparatus and are uncorrected. Optical rotations were measured with a Perkin-Elmer 343 polarimeter. NMR 
spectra were recorded with a Bruker AC 200 spectrometer at $200 \mathrm{MHz}\left({ }^{1} \mathrm{H}\right)$ and $50.3 \mathrm{MHz}\left({ }^{13} \mathrm{C}\right)$ or with a Bruker AM 500 spectrometer at $500 \mathrm{MHz}\left({ }^{1} \mathrm{H}\right)$ and $125 \mathrm{MHz}\left({ }^{13} \mathrm{C}\right)$. Homo and heteronuclear correlation spectroscopy experiments were performed when indicated. The two branching Galp were indistinctly numbered with prime or double prime.

\section{5,6-O-Isopropylidene-2,3-di-O-(2,3,4,6-tetra- $O$-acetyl- $\beta$-D-galactopyranosyl)-D-galactono-} 1,4-lactone (3). To a flask containing recently purified and dried 5,6-O-isopropylidene-Dgalactono-1,4-lactone $^{12}(\mathbf{1}, 0.55 \mathrm{~g}, 2.56 \mathrm{mmol})$ and activated $4 \AA$ powdered molecular sieves, was added a solution of $O$-(2,3,4,6-tetra-O-acetyl- $\alpha$-D-galactopyranosyl) trichloroacetimidate ${ }^{14}$ $(2,3.00 \mathrm{~g}, 6.10 \mathrm{mmol})$ in freshly distilled anhydrous $\mathrm{CH}_{2} \mathrm{Cl}_{2}(50 \mathrm{~mL})$ and the suspension was cooled to $-20^{\circ} \mathrm{C}$. After $15 \mathrm{~min}$ of vigorous stirring, TMSOTf $(184 \mu \mathrm{l}, 1.01 \mathrm{mmol})$ was slowly added and the stirring continued for $1 \mathrm{~h}$ until TLC examination showed consumption of imidate 2. The reaction was quenched by addition of triethylamine $(132 \mu \mathrm{l}, 1.01 \mathrm{mmol})$ and the mixture was allowed to reach room temperature and then filtered over Celite. The filtrate was concentrated and the residue was purified by column chromatography ( $7: 3$ toluene-EtOAc) to give $1.85 \mathrm{~g}$ of foamy solid that crystallized from 3:1 hexane-isopropanol.The product was characterized as 3 (82 \%): $\mathrm{R}_{f} 0.55$ (1:2 toluene-EtOAc), $[\alpha]_{\mathrm{D}}-16.6^{\circ}\left(\mathrm{c} 1, \mathrm{CHCl}_{3}\right)$; mp 102$105^{\circ} \mathrm{C} ;{ }^{1} \mathrm{H}$ NMR $\left(\mathrm{CDCl}_{3}, 500 \mathrm{MHz}\right): \delta 5.44\left(\mathrm{dd}, 1 \mathrm{H}, J=3.4,1.3 \mathrm{~Hz}, \mathrm{H}-4^{\prime \prime}\right), 5.42(\mathrm{dd}, 1 \mathrm{H}, J=$ 3.4, $1.1 \mathrm{~Hz}, \mathrm{H}-4^{\prime}$ ), 5.22 (dd, 1H, $\left.J=7.8,10.3 \mathrm{~Hz}, \mathrm{H}-2^{\prime \prime}\right), 5.18$ (dd, $1 \mathrm{H}, J=7.9,10.4 \mathrm{~Hz}, \mathrm{H}-2^{\prime}$ ), 5.09 (dd, $1 \mathrm{H}, J=10.4,3.4 \mathrm{~Hz}, \mathrm{H}-3^{\prime}$ ), 5.04 (dd, 1H, $\left.J=10.3,3.4 \mathrm{~Hz}, \mathrm{H}-3^{\prime \prime}\right), 4.79$ (dd, $1 \mathrm{H}, J=7.7$, $6.6 \mathrm{~Hz}, \mathrm{H}-3$ ), 4.73 (d, 1H, J = 7.9 Hz, H-1'), 4.72 (d, 1H, $\left.J=7.8 \mathrm{~Hz}, \mathrm{H}-1^{\prime \prime}\right), 4.60$ (d, 1H, J= 7.7 Hz, H-2), 4.40 (ddd, 1H, J = 2.3, 6.6, 6.8 Hz, H-5), 4.25 (dd, 1H, $J=6.1,11.5$ Hz, H-6"a), 4.25 (dd, $1 \mathrm{H}, J=6.6,2.3 \mathrm{~Hz}, \mathrm{H}-4), 4.17$ (dd, $1 \mathrm{H}, J=6.8,11.3 \mathrm{~Hz}, \mathrm{H}-6$ ' a), 4.16 (dd, $1 \mathrm{H}, J=6.8,11.5$ Hz, H-6"b), 4.12 (dd, 1H, J = 6.3, $11.3 \mathrm{~Hz}, \mathrm{H}-6^{\prime}$ b), 4.10 (dd, 1H, J = 6.8, 8.7 Hz, H-6a), 3.98 (dd, $1 \mathrm{H}, J=6.6,8.7 \mathrm{~Hz}, \mathrm{H}-6 \mathrm{~b}), 3.98$ (m, 1H, H-5'), 3.92 (m, 1H, H-5"), 2.17, 2.16, 2.13, 2.12, 2.07, 2.06, 2.00, $1.99\left(8 \mathrm{~s}, 24 \mathrm{H}, \mathrm{CH}_{3} \mathrm{CO}\right), 1.41,1.38\left(2 \mathrm{~s}, 6 \mathrm{H},\left(\mathrm{CH}_{3}\right)_{2} \mathrm{C}\right) ;{ }^{13} \mathrm{C} \mathrm{NMR}\left(\mathrm{CDCl}_{3}, 125 \mathrm{MHz}\right)$ : $\delta$ 170.3, 170.2, 170.1, 170.0, 169.9, 169.7, $169.5\left(\mathrm{CH}_{3} \mathrm{CO}\right), 168.3(\mathrm{C}-1), 110.3\left(\left(\mathrm{CH}_{3}\right)_{2} \mathrm{C}\right), 101.0$ (C-1"), 99.4 (C-1'), 79.8 (C-3), 79.1 (C-2), 78.5 (C-4), 73.6 (C-5), 71.3 (C-5"), 70.9 (C-5'), 70.4 (C-3', C-3"), 68.7 (C-2'), 68.5 (C-2"), 66.8, 66.7 (C-4', C-4"), 65.1 (C-6), 61.3, 61.1 (C-6', C-6"), 25.9, $25.7\left(\left(\mathrm{CH}_{3}\right)_{2} \mathrm{C}\right), 20.8,20.7,20.6,20.5\left(\mathrm{CH}_{3} \mathrm{CO}\right)$. The assignments were supported by homo and heteronuclear correlation spectroscopy experiments.

Anal. Calcd for $\mathrm{C}_{37} \mathrm{H}_{50} \mathrm{O}_{24 .:}$ C, 50.57; H, 5.73 . Found: C, 50.38; H, 5.68.

\section{5,6-O-Isopropylidene-2,3-di-O-(2,3,4,6-tetra- $O$-acetyl- $\beta$-D-galactopyranosyl)-D-galactofuranose}

(4). A freshly solution of bis(2-butyl-3-methyl)borane in anhydrous THF was prepared from 2,2dimethylbutene $(2.8 \mathrm{ml}, 23.9 \mathrm{mmol})$ and $3.4 \mathrm{M} \mathrm{BH}_{3}$ in THF $(3.5 \mathrm{ml}, 11.95 \mathrm{mmol})$ cooled at $0{ }^{\circ} \mathrm{C}$. This solution was cannula-added to a flask containing dried $3(1.75 \mathrm{~g}, 1.99 \mathrm{mmol})$ under an argon atmosphere. After $3 \mathrm{~h}$, dissolution was total and the stirring continued for additional $45 \mathrm{~h}$ until TLC examination showed consumption of lactone 3 . The reaction was quenched with water, and then $30 \% \mathrm{H}_{2} \mathrm{O}_{2}$ maintaining the $\mathrm{pH} 6-8$ with $2.5 \mathrm{M} \mathrm{KOH}$ as already described. ${ }^{15}$ 
After addition of water $(15 \mathrm{~mL})$, the mixture was extracted with $\mathrm{CH}_{2} \mathrm{Cl}_{2}(3 \mathrm{x} 80 \mathrm{~mL})$. The organic phase was washed with water, dried $\left(\mathrm{Na}_{2} \mathrm{SO}_{4}\right)$, and concentrated. Boric acid was removed by careful co-evaporation of the syrup with methanol at room temperature. The residue was purified by column chromatography (1:1 toluene-EtOAc). Compound 4 (1.0 g, 57 \%) eluted first $\left(\mathrm{R}_{f}\right.$ 0.5, 1:3 toluene-EtOAc) as an amorphous solid, that crystallized from 9:1 hexaneisopropanol: $\mathrm{mp} 95-100{ }^{\circ} \mathrm{C},[\alpha]_{\mathrm{D}}-14.5^{\circ}\left(\mathrm{c} 1, \mathrm{CHCl}_{3}\right) ;{ }^{1} \mathrm{H} \mathrm{NMR}\left(\mathrm{CDCl}_{3}, 500 \mathrm{MHz}\right): \delta$ ( $\delta$ of the $\beta$ anomer are listed, only few signals of the $\alpha$ anomer are shown for integration purposes) 5.46 (dd, $0.75 \mathrm{H}, J=2.5,4.3 \mathrm{~Hz}, \mathrm{H}-1 \beta), 5.41$ (dd, 0.75H, $\left.J=3.6,1.1 \mathrm{~Hz}, \mathrm{H}-4{ }^{\prime \prime}\right), 5.38$ (dd, 0.75H, $J=3.4$, $\left.1.3 \mathrm{~Hz}, \mathrm{H}-4^{\prime}\right), 5.22$ (dd, 0.75H, J = 7.9, $\left.10.4 \mathrm{~Hz}, \mathrm{H}-2^{\prime \prime}\right), 5.15$ (dd, 0.75H, J = 7.9, $10.4 \mathrm{~Hz}, \mathrm{H}-2^{\prime}$ ), 5.06 (dd, 0.75H, $\left.J=10.4,3.4 \mathrm{~Hz}, \mathrm{H}-3^{\prime}\right), 5.05$ (dd, 0.75H, $\left.J=10.4,3.6 \mathrm{~Hz}, \mathrm{H}-3^{\prime \prime}\right), 4.60,459$ (2d, $1.5 \mathrm{H}, J=7.9 \mathrm{~Hz}, \mathrm{H}-1^{\prime}$ and $\left.\mathrm{H}-1^{\prime \prime}\right), 4.61$ (d, $0.25 \mathrm{H}, J=7.9 \mathrm{~Hz}, \mathrm{H}-1^{\prime}$ or H-1" from $\alpha$ anomer), 4.57 (d, 0.25H, $J=7.7 \mathrm{~Hz}, \mathrm{H}-1^{\prime}$ or H-1" from $\alpha$ anomer), 4.23-4.11 (m), $4.03(\mathrm{dd}, 0.75 \mathrm{H}, J=2.5,4.5$ $\mathrm{Hz}), 4.00(\mathrm{dd}, 0.75 \mathrm{H}, J=6.7,8.7 \mathrm{~Hz}), 3.97-3.92(\mathrm{~m}, 1.5 \mathrm{H}), 3.87(\mathrm{dd}, 0.75 \mathrm{H}, J=6.7,8.7 \mathrm{~Hz})$, 3.55 (d, 0.25H, $J=6.0 \mathrm{~Hz}, \mathrm{OH}-\alpha), 3.21(\mathrm{~d}, 0.75 \mathrm{H}, J=4.3 \mathrm{~Hz}, \mathrm{OH}-\beta), 2.17,2.16,2.11,2.08$, 2.07, 1.99, $1.98\left(8 \mathrm{~s}, \mathrm{CH}_{3} \mathrm{CO}\right), 1.43,1.37\left(2 \mathrm{~s}, 4.5 \mathrm{H},\left(\mathrm{CH}_{3}\right)_{2} \mathrm{C}\right), 1.46,1.39\left(2 \mathrm{~s}, 1.5 \mathrm{H},\left(\mathrm{CH}_{3}\right)_{2} \mathrm{C}\right.$ from $\alpha$ anomer). ${ }^{13} \mathrm{C} \mathrm{NMR}\left(\mathrm{CDCl}_{3}, 50.3 \mathrm{MHz}\right): \delta$ for the $\beta$ anomer: $170.4,170.2,170.1,170.0,169.5$, $169.2\left(\mathrm{CH}_{3} \mathrm{CO}\right), 109.6\left(\left(\mathrm{CH}_{3}\right)_{2} \mathrm{C}\right), 100.6,100.5,99.7$ (C-1', C-1", C-1 $\left.\beta\right), 94.5(\mathrm{C}-1 \alpha), 89.4(\mathrm{C}-2)$, 81.3 ,80.2 (C-3, C-4), 75.4, 71.0, 70.7, 70.6, 70.4; 68.9, 68.8 (C-2', C-2"); 66.9 (C-4', C-4"), 65.2 (C-6), 61.7, $61.1\left(\mathrm{C}-6^{\prime}, \mathrm{C}-6^{\prime \prime}\right), 26.2,25.3\left(\left(\mathrm{CH}_{3}\right)_{2} \mathrm{C}\right), 20.8,20.7,20.6,20.5\left(\mathrm{CH}_{3} \mathrm{CO}\right)$. Anal. Calcd for $\mathrm{C}_{37} \mathrm{H}_{52} \mathrm{O}_{24}$ : C, 50.45; H, 5.95. Found: $\mathrm{C}, 50.41, \mathrm{H}, 6.04$.

Next fraction of the column $\left(\mathrm{R}_{f} 0.3,1: 3\right.$ toluene-EtOAc) afforded $0.20 \mathrm{~g}$ of a foamy compound which was characterized as 2,3-di-O-(2,3,4,6-tetra-O-acetyl- $\beta$-D-galactopyranosyl)D-galactono-1,4-lactone (5) (15\%): $[\alpha]_{\mathrm{D}}+0.6^{\circ}\left(c\right.$ 1, $\left.\mathrm{CHCl}_{3}\right) ;{ }^{1} \mathrm{H} \mathrm{NMR}\left(\mathrm{CDCl}_{3}, 500 \mathrm{MHz}\right): \delta$ 5.42 (dd, $1 \mathrm{H}, J=2.5,1.5 \mathrm{~Hz}, \mathrm{H}-4^{\prime}$ or H-4"), 5.41 (dd, 1H, $J=2.4,1.3 \mathrm{~Hz}, \mathrm{H}-4^{\prime}$ or H-4"), 5.20 (dd, $\left.1 \mathrm{H}, J=7.6,10.2 \mathrm{~Hz}, \mathrm{H}-2^{\prime \prime}\right), 5.18\left(\mathrm{dd}, 1 \mathrm{H}, J=7.9,10.4 \mathrm{~Hz}, \mathrm{H}-2^{\prime}\right), 5.07$ (dd, $1 \mathrm{H}, J=10.4,3.4 \mathrm{~Hz}$, H-3'), 5.05 (dd, 1H, $\left.J=10.2,3.6 \mathrm{~Hz}, \mathrm{H}-3^{\prime \prime}\right), 4.90$ (dd, 1H, $\left.J=8.3,7.1 \mathrm{~Hz}, \mathrm{H}-3\right), 4.78$ (d, $1 \mathrm{H}, J=$ $\left.7.6 \mathrm{~Hz}, \mathrm{H}-1^{\prime \prime}\right), 4.73$ (d, 1H, J=7.9 Hz, H-1'), 4.63 (d, 1H, J=8.3 Hz, H-2), 4.33 (dd, 1H, J= 7.1, $2.2 \mathrm{~Hz}, \mathrm{H}-4), 4.21-4.13(\mathrm{~m}, 5 \mathrm{H}), 4.05-4.01$ (m, 1H), 3.98, 3.92 (2m, 2H, H-5'and H-5"), 3.823.71 (m, 2H, H-6a,6b), 3.03 (d, 1H, J = 7.6 Hz, OH), 2.18, 2.17, 2.13, 2.12, 2.09, 2.08, 2.00, 1.99 $\left(8 \mathrm{~s}, 24 \mathrm{H}, \mathrm{CH}_{3} \mathrm{CO}\right) ;{ }^{13} \mathrm{C} \mathrm{NMR}\left(\mathrm{CDCl}_{3}, 125 \mathrm{MHz}\right): \delta 170.7,170.6,170.1,170.0,169.9,169.7$, $169.6\left(\mathrm{CH}_{3} \mathrm{CO}\right), 168.9$ (C-1), 101.4, 99.0 (C-1', C-1"), 80.1, 79.9, 78.6 (C-4, C-3, C-2), 71.5, 71.3, 70.4 (x2), 68.8, 68.7, 67.1, 67.0, 63.4 (C-6), 61.8, 61.7 (C-6', C-6"'), 20.8, 20.7, 20.6, 20.5 $\left(\mathrm{CH}_{3} \mathrm{CO}\right)$.

Last fraction of the column $\left(\mathrm{R}_{f} 0.1,1: 3\right.$ toluene-EtOAc) gave $0.24 \mathrm{~g}$ of 2,3-di-O-(2,3,4,6-tetra$O$-acetyl- $\beta$-D-galactopyranosyl)-D-galactose (6) (14\%) as a foamy solid that crystallized from 8:2 hexane-EtOAc, as a mixture of anomers $\left(\mathrm{R}_{f} 0.28,0.18\right.$ and 0.11 , EtOAc): $\mathrm{mp} 115-125^{\circ} \mathrm{C}$, $[\alpha]_{\mathrm{D}}-3.7^{\mathrm{o}}\left(\mathrm{c} 1, \mathrm{CHCl}_{3}\right) ;{ }^{13} \mathrm{C} \mathrm{NMR}\left(\mathrm{CDCl}_{3}, 125 \mathrm{MHz}\right): \delta$ anomeric region 101.5, 101.1, 101.0, $100.8,100.7,100.6,100.5$ (C-1'and C-1" of each anomer, and C-1 $\beta$ furanosic), 95.5 (C-1 $\alpha$ furanosic), 94.8 (C-1 $\beta$ pyranosic), 92.5 (C-1 $\alpha$ pyranosic).

Anal. Calcd for $\mathrm{C}_{34} \mathrm{H}_{48} \mathrm{O}_{24}$ : C, 48.57; H, 5.75. Found: C, 48.12; H, 5.84. 
2,3-Di-O-(2,3,4,6-tetra- $O$-acetyl- $\beta$-D-galactopyranosyl)-D-galactose (6). To a solution of 5,6$O$-isopropylidene-2,3-di-O-(2,3,4,6-tetra- $O$-acetyl- $\beta$-D-galactopyranosyl)-D-galactofuranose (4, $0.73 \mathrm{~g}, 0.83 \mathrm{mmol})$ in acetic acid $(4.2 \mathrm{~mL})$ warmed at $60^{\circ} \mathrm{C}$, was added $\mathrm{H}_{2} \mathrm{O}(1.5 \mathrm{~mL})$ slowly with stirring. After $30 \mathrm{~min}$, the mixture was cooled, concentrated under vacuum and the acetic acid was eliminated by successive coevaporations with water and then, toluene to give $0.69 \mathrm{~g}$ of $6(98 \%)$ with the same properties as described above.

1,5,6-Tri-O-acetyl-2,3-di-O-(2,3,4,6-tetra- $O$-acetyl- $\beta$-D-galactopyranosyl)-Dgalactofuranose (7f) and 1,4,6-tri- $O$-acetyl - 2,3-di- $O$ - (2,3,4,6- tetra - $O$-acetyl - $\beta$ - D galactopyranosyl)-D-galactopyranose (7p). To a stirred solution of 2,3-di-O-(2,3,4,6-tetra-Oacetyl- $\beta$-D-galactopyranosyl)-D-galactose $(6,0.69 \mathrm{~g}, 0.82 \mathrm{mmol})$ in dry pyridine $(3.8 \mathrm{ml})$ at $15^{\circ} \mathrm{C}$, acetic anhydride $(3 \mathrm{ml})$ was added dropwise over $45 \mathrm{~min}$, and the mixture was stirred for 13 $\mathrm{h}$ at $-18{ }^{\circ} \mathrm{C}$. After $6 \mathrm{~h}$ at $5{ }^{\circ} \mathrm{C}$, TLC monitoring of the crude reaction mixture showed two main spots of $\mathrm{Rf} 0.58,0.53$ (toluene-EtOAc 1:3). The mixture was cooled to $0{ }^{\circ} \mathrm{C}, \mathrm{MeOH}(5 \mathrm{ml}$ ) was added and the stirring continued for $30 \mathrm{~min}$. The solution was diluted with $\mathrm{CH}_{2} \mathrm{Cl}_{2}(150 \mathrm{ml})$, and washed with $10 \% \mathrm{HCl}(2 \times 40 \mathrm{ml})$, water $(40 \mathrm{ml})$, saturated aqueous $\mathrm{NaHCO}_{3}(40 \mathrm{ml})$ and water $(80 \mathrm{ml})$. The organic phase was dried $\left(\mathrm{MgSO}_{4}\right)$, filtered and concentrated to give $0.72 \mathrm{~g}$ of a foamy solid (91\%). ${ }^{1} \mathrm{H} \mathrm{NMR}$ spectrum $\left(\mathrm{CDCl}_{3}, 500 \mathrm{MHz}\right)$ showed the anomeric signals with the following integration $\delta 6.36(\mathrm{~d}, 0.4 \mathrm{H}, J=3.8 \mathrm{~Hz}, \mathrm{H}-1 \alpha$ pyranosic), 6.31 (bs, $0.34 \mathrm{H}, \mathrm{H}-1 \beta$ furanosic), $6.17(\mathrm{~d}, 0.20 \mathrm{H}, J=4.2 \mathrm{~Hz}, \mathrm{H}-1 \alpha$ furanosic $), 5.63(\mathrm{~d}, 0.06 \mathrm{H}, J=7.6 \mathrm{~Hz}, \mathrm{H}-1 \beta$ pyranosic). The crude mixture was partially separated by column chromatography $(9: 2$ tolueneEtOAc) to give a first fraction of $0.33 \mathrm{~g}$ which contained mainly the $\alpha$ and $\beta$ furanosic products 7f $(\mathrm{R} f$ 0.58, $42 \%),{ }^{1} \mathrm{H} \mathrm{NMR}\left(\mathrm{CDCl}_{3}, 200 \mathrm{MHz}\right): \delta$ anomeric region $6.31(\mathrm{bs}, 0.6 \mathrm{H}, \mathrm{H}-1 \beta f), 6.17$ $(\mathrm{d}, 0.4 \mathrm{H}, J=4.2 \mathrm{~Hz}, \mathrm{H}-1 \alpha f) .{ }^{13} \mathrm{C} \mathrm{NMR}\left(\mathrm{CDCl}_{3}, 50.3 \mathrm{MHz}\right): \delta$ anomeric region 101.5, 101.3, 100.1, 99.7, 99.4 (C-1', C-1" of each anomer and C-1 $\beta f), 93.0$ (C-1 $\alpha f)$.

A second fraction ( $R f 0.53)$ was characterized as the pyranosic product $7 \mathbf{p}(0.29 \mathrm{~g}, 29 \%)$ that crystallized from 2:1 hexane-ether. Compound $7 \mathbf{p}$ gave: $\mathrm{mp} 103-107^{\circ} \mathrm{C},[\alpha]_{\mathrm{D}}+19.2^{\circ}$ (c 1 , $\left.\mathrm{CHCl}_{3}\right) ;{ }^{1} \mathrm{H}$ NMR $\left(\mathrm{CDCl}_{3}, 500 \mathrm{MHz}\right): \delta 6.36(\mathrm{~d}, 1 \mathrm{H}, J=3.8 \mathrm{~Hz}, \mathrm{H}-1), 5.45(\mathrm{~d}, 1 \mathrm{H}, J=3.3 \mathrm{~Hz}$, H-4), 5.35 (d, 1H, $\left.J=3.4 \mathrm{~Hz}, \mathrm{H}-4^{\prime}\right), 5.33$ (d, 1H, $\left.J=3.6, \mathrm{H}-4^{\prime \prime}\right), 5.14$ (dd, 1H, $J=7.7,10.3 \mathrm{~Hz}$, H-2'), 5.08 (dd, 1H, J = 7.5, 10.5 Hz, H-2"), 4.99 (dd, 1H, J = 10.3, 3.4 Hz, H-3'), 4.97 (dd, 1H, $J$ $\left.=10.5,3.6 \mathrm{~Hz}, \mathrm{H}-3^{\prime \prime}\right), 4.63$ (d, 1H, $\left.J=7.5 \mathrm{~Hz}, \mathrm{H}-1^{\prime \prime}\right), 4.57$ (d, 1H, J = 7.7 Hz, H-1'), 4.23 (dd, $1 \mathrm{H}, J=6.0,11.2 \mathrm{~Hz}), 4.15-3.87(\mathrm{~m}, 10 \mathrm{H}), 2.18,2.16,2.15,2.14,2.12,2.08,2.07,2.06,2.04$, 1.98, $1.97\left(11 \mathrm{~s}, 33 \mathrm{H}, \mathrm{CH}_{3} \mathrm{CO}\right) ;{ }^{13} \mathrm{C} \mathrm{NMR}\left(\mathrm{CDCl}_{3}, 50.3 \mathrm{MHz}\right): \delta 170.5,170.4,170.3,170.1$, 170.0, 169.9, 169.6, 169.5, 168.9, $168.7\left(\mathrm{CH}_{3} \mathrm{CO}\right), 101.4,100.4$ (C-1', C-1"), 91.3 (C-1), 75.3, 72.0, 70.8, 70.7, 70.6, 70.2, 69.8, 69.7, 69.6, 68.6; 66.8, 66.7 (C-4', C-4"); 62.1 (C-6), 61.1, 60.9 (C-6', C-6"), 21.0, 20.9 , 20.7, 20.6, $20.5\left(\mathrm{CH}_{3} \mathrm{CO}\right)$.

Anal. Calcd for $\mathrm{C}_{40} \mathrm{H}_{54} \mathrm{O}_{27}$ : C, 49.69; H, 5.63. Found: C, 49.49; H, 5.70.

4,6-Di-O-Acetyl-2,3-di-O-(2,3,4,6-tetra-O-acetyl- $\beta$-D-galactopyranosyl)-D-galactopyranose (8) from 1,4,6-tri-O-acetyl-2,3-di-O-(2,3,4,6-tetra-O-acetyl- $\beta$-D-galactopyranosyl)-Dgalactopyranose (7p). To a cooled solution $\left(0^{\circ} \mathrm{C}\right)$ of glacial acetic acid (13 $\left.\mu \mathrm{l}\right)$ in THF $(2.9 \mathrm{ml})$ 
ethylenediamine $(13 \mu \mathrm{l}, 0.19 \mathrm{mmol})$ was added with stirring. After $10 \mathrm{~min}, 1,4,6$-tri-O-acetyl2,3-di-O-(2,3,4,6-tetra-O-acetyl- $\beta$-D-galactopyranosyl)-D-galactopyranose (7p, $124.5 \mathrm{mg}, 0.129$ mmol) was added and the solution was stirred for $20 \mathrm{~h}$ at room temperature. The mixture was diluted with $\mathrm{CH}_{2} \mathrm{Cl}_{2}(50 \mathrm{ml})$, washed with water $(10 \mathrm{ml}), 10 \% \mathrm{HCl}(10 \mathrm{ml})$, water $(30 \mathrm{ml})$; saturated aqueous $\mathrm{NaHCO}_{3}(20 \mathrm{ml})$, and water $(2 \times 30 \mathrm{ml})$. The organic phase was dried $\left(\mathrm{MgSO}_{4}\right)$, filtered and evaporated. Purification of the residue by column chromatography $3: 7$ toluene-EtOAc) gave $98 \mathrm{mg}$ of 8 in the $\alpha$-configuration at the free anomeric carbon, as an amorphous syrup that crystallized from 10:2:1 hexane-ether-isopropanol (80\%): $\mathrm{R} f$ 0.35 (3:7 toluene-EtOAc), mp $115-118^{\circ} \mathrm{C}$. Isomerization in a chloroformic solution for a week gave a 9:1 $\alpha / \beta$ mixture: $[\alpha]_{\mathrm{D}}+11.4^{\circ}\left(c 1, \mathrm{CHCl}_{3}\right) .{ }^{1} \mathrm{H} \mathrm{NMR}\left(\mathrm{CDCl}_{3}, 500 \mathrm{MHz}\right): \delta(\delta$ for the $\alpha$ anomer are listed, only the H-2 signal of the $\beta$ anomer is shown for integration purposes) 5.43 (dd, $0.9 \mathrm{H}, J=$ $3.6,1.5 \mathrm{~Hz}), 5.38(\mathrm{~m}, 2 \mathrm{H}), 5.34(\mathrm{dd}, 0.9 \mathrm{H}, J=3.4,1.3), 5.24$ (dd, 0.9H, $\left.J=7.9,10.6 \mathrm{~Hz}, \mathrm{H}-2^{\prime}\right)$, $5.08\left(\mathrm{dd}, 0.9 \mathrm{H}, J=7.5,10.6 \mathrm{~Hz}, \mathrm{H}-2^{\prime \prime}\right), 5.02(\mathrm{dd}, 0.9 \mathrm{H}, J=10.6,3.4 \mathrm{~Hz}), 5.00(\mathrm{dd}, 0.9 \mathrm{H}, J=$ 10.6, 3.6 Hz), 4.65 (d, 0.9H, $\left.J=7.5 \mathrm{~Hz}, \mathrm{H}-1^{\prime \prime}\right), 4.59$ (d, 0.9H, $J=7.9 \mathrm{~Hz}, \mathrm{H}-1^{\prime}$ ), 4.37 (m, 0.9H, H-5), 4.23 (dd, 0.9H, $J=9.7,3.6 \mathrm{~Hz}, \mathrm{H}-3), 4.22$ (dd, 0.9H, $J=7.0,11.5 \mathrm{~Hz}), 4.18$ (dd, $0.9 \mathrm{H}, J=$ 7.0, $11.3 \mathrm{~Hz}), 4.14(\mathrm{dd}, 0.9 \mathrm{H}, J=7.0,11.3 \mathrm{~Hz}), 4.12(\mathrm{dd}, 0.9 \mathrm{H}, J=5.4,11.7 \mathrm{~Hz}), 4.09(\mathrm{dd}, 0.9 \mathrm{H}$, $J=5.9,11.5 \mathrm{~Hz}), 4.02(\mathrm{dd}, 0.9 \mathrm{H}, J=7.2,11.5 \mathrm{~Hz}), 3.93\left(\mathrm{~m}, 0.9 \mathrm{H}, \mathrm{H}-5^{\prime}\right.$ or H-5"), 3.90 (dd, 0.9H, $J=9.7,3.2 \mathrm{~Hz}, \mathrm{H}-2), 3.87$ (m, 0.9H, H-5' or H-5"), 3.68 (dd, 0.1H, $J=7.5,9.5 \mathrm{~Hz}, \mathrm{H}-2$ $\beta$ anomer $), 2.18,2.17,2.13,2.12,2.08,2.07,2.06,1.99,1.97\left(10 \mathrm{~s}, \mathrm{CH}_{3} \mathrm{CO}\right) ;{ }^{13} \mathrm{C} \mathrm{NMR}\left(\mathrm{CDCl}_{3}\right.$, $125 \mathrm{MHz}): \delta$ of $\alpha$ anomer 170.6, 170.5, 170.3, 170.1, 169.9, 169.7, 169.6, $168.9\left(\mathrm{CH}_{3} \mathrm{CO}\right)$, 101.7, 100.2 (C-1', C-1"), 95.5 (C-1 $\beta$ anomer), 92.4 (C-1 $\alpha$ anomer), 78.3, 71.4, 71.2, 70.8, 70.7, 70.3, 70.2, 69.7, 69.3, 67.2, 67.0, 66.9; 62.4 (C-6), 61.3, 61.0 (C-6', C-6"), 21.0, 20.9, 20.8, 20.6, 20.5, $20.4\left(\mathrm{CH}_{3} \mathrm{CO}\right)$.

Anal. Calcd $\mathrm{C}_{38} \mathrm{H}_{52} \mathrm{O}_{26}$ : C, 49.35; H, 5.67. Found: C, 49.17; H, 5.76.

From benzyl 4,6-di-O-acetyl-2,3-di-O-(2,3,4,6-tetra-O-acetyl- $\beta$-D-galactopyranosyl)- $\beta$-Dgalactopyranoside (12). To a solution of 12 (200 mg, $0.197 \mathrm{mmol})$ in EtOAc (4.5 mL), was added 10\% Pd/C Deguzza type E101 NE/W (100 mg), and the suspension was hydrogenated $2 \mathrm{~h}$ at $1.5 \mathrm{~atm}$ and $33^{\circ} \mathrm{C}$. The catalyst was filtered and the filtrate was concentrated to give $178 \mathrm{mg}$ of compound $8(98 \%)$ in the $\beta$-configuration at the free anomeric carbon. Isomerization of the compound in a chloroformic solution for a week gave a $3: 1 \beta / \alpha$ mixture: $[\alpha]_{\mathrm{D}}+6.7^{\circ}$ (c 1 , $\left.\mathrm{CHCl}_{3}\right) .{ }^{1} \mathrm{H} \mathrm{NMR}\left(\mathrm{CDCl}_{3}, 500 \mathrm{MHz}\right): \delta$ ( $\delta$ for the $\beta$ anomer are listed, only the $\mathrm{H}-5$ signal of the $\alpha$ anomer is shown for integration purposes) 5.41 (dd, $0.75 \mathrm{H}, J=3.8,0.9 \mathrm{~Hz}, \mathrm{H}-4), 5.38$ (dd, $\left.0.75 \mathrm{H}, J=3.5,1.1 \mathrm{~Hz}, \mathrm{H}-4^{\prime \prime}\right), 5.36\left(\mathrm{dd}, 0.75 \mathrm{H}, J=3.4,1.1 \mathrm{~Hz}, \mathrm{H}-4^{\prime}\right), 5.26$ (dd, 0.75H, $J=7.9$, $10.5 \mathrm{~Hz}, \mathrm{H}-2^{\prime}$ ), 5.09 (dd, 0.75H, $\left.J=7.5,10.7 \mathrm{~Hz}, \mathrm{H}-2^{\prime \prime}\right), 5.00$ (dd, 0.75H, $J=10.5,3.4 \mathrm{~Hz}, \mathrm{H}-3^{\prime}$ ), 4.99 (dd, 0.75H, $\left.J=10.7,3.5 \mathrm{~Hz}, \mathrm{H}-3^{\prime \prime}\right), 4.67$ (dd, 0.75H, $\left.J=4.3,7.5 \mathrm{~Hz}, \mathrm{H}-1\right), 4.65$ (d, 0.75H, $\left.=7.5 \mathrm{~Hz}, \mathrm{H}-1^{\prime \prime}\right), 4.63\left(\mathrm{~d}, 0.75 \mathrm{H}, J=7.9 \mathrm{~Hz}, \mathrm{H}-1^{\prime}\right), 4.43(\mathrm{~d}, 0.75 \mathrm{H}, J=4.3 \mathrm{~Hz}, \mathrm{OH}-), 4.37$ (m, $0.25 \mathrm{H}, \mathrm{H}-5 \alpha$ anomer), 4.20-4.12 (m), $4.05(\mathrm{dd}, 0.75 \mathrm{H}, J=7.2,11.7 \mathrm{~Hz}), 3.98(\mathrm{~m}, 0.75 \mathrm{H}), 3.89$ $(\mathrm{dd}, 0.75 \mathrm{H}, J=9.5,3.8 \mathrm{~Hz}, \mathrm{H}-3), 3.85(\mathrm{~m}, 0.75 \mathrm{H}), 3.80(\mathrm{~m}, 0.75 \mathrm{H}), 3.68(\mathrm{dd}, 0.75 \mathrm{H}, J=7.5,9.5$ $\mathrm{Hz}, \mathrm{H}-2$ ), 2.18, 2.17, 2.14, 2.13, 2.10, 2.09, 2.07, 2.06, 1.99, $1.98\left(10 \mathrm{~s}, 30 \mathrm{H}, \mathrm{CH}_{3} \mathrm{CO}\right) ;{ }^{13} \mathrm{C} \mathrm{NMR}$ $\left(\mathrm{CDCl}_{3}, 125 \mathrm{MHz}\right): \delta 170.6,170.4,170.3,170.2,170.1,170.0,169.8,169.4,169.3,169.1$ 
$\left(\mathrm{CH}_{3} \mathrm{CO}\right), 100.9,100.5$ (C-1', C-1"), 95.5 (C-1 $\left.\beta\right), 92.4$ (C-1 $\left.\alpha\right), 80.5,76.1$ (C-2, C-3), 71.4 (x2), 70.9, 70.6, 70.3, 69.7 (x2), 69.2, 66.9; 62.4 (C-6); 61.3, 61.2 (C-6', C-6"), 20.9, 20.8, 20.7, 20.6, 20.5, $20.4\left(\mathrm{CH}_{3} \mathrm{CO}\right)$.

Benzyl 4,6-O-benzylidene-2,3-di-O-(2,3,4,6-tetra-O-acetyl - $\beta$ - D - galactopyranosyl) - $\beta$-D galactopyranoside (10). A suspension of dried benzyl 4,6 - O - benzylidene - $\beta-D$ galactopyranoside $^{18}(\mathbf{9}, 0.97 \mathrm{~g}, 2.71 \mathrm{mmol}), O$-(2,3,5,6-tetra-O-acetyl- $\beta$-D-galactopyranosyl) tricloroacetimidate $^{14}(2,3.34 \mathrm{~g}, 6.78 \mathrm{mmol})$, activated $4 \AA$ powdered molecular sieves in anhydrous $\mathrm{CH}_{2} \mathrm{Cl}_{2}(130 \mathrm{~mL})$ was stirred under an argon atmosphere at $-15^{\circ} \mathrm{C}$ for 15 min and TMSOTf (0.4 equiv, $196 \mu \mathrm{l}$ ) was slowly added. After stirring for $1 \mathrm{~h}$, TLC examination showed consumption of $9(\mathrm{R} f 0.15$, toluene-EtOAc $1: 2)$. Triethylamine (151 $\mu \mathrm{l})$ was slowly added and the mixture was allowed to reach room temperature. The suspension was filtered, the solid washed with $\mathrm{CH}_{2} \mathrm{Cl}_{2}$ and the filtrate was concentrated under vacuum. Purification of the residue by silica gel column chromatography (3:2 toluene-EtOAc) yielded $1.65 \mathrm{~g}$ of pure $10(59 \%, \mathrm{R} f$ 0.45 , toluene-EtOAc 1:2) which crystallized from isopropanol: $\mathrm{mp} 115-117^{\circ} \mathrm{C},[\alpha]_{\mathrm{D}}-17.2^{\circ}(\mathrm{c}$ $\left.1, \mathrm{CHCl}_{3}\right) ;{ }^{1} \mathrm{H}$ NMR $\left(\mathrm{CDCl}_{3}, 500 \mathrm{MHz}\right): \delta$ 7.55-7.25 (m, $\left.10 \mathrm{H}\right), 5.53(\mathrm{~s}, 1 \mathrm{H}, \mathrm{PhCH}), 5.37(\mathrm{dd}$, $\left.1 \mathrm{H}, J=3.4,1.1 \mathrm{~Hz}, \mathrm{H}-4^{\prime}\right), 5.29$ (dd, $\left.1 \mathrm{H}, J=3.4,1.1 \mathrm{~Hz}, \mathrm{H}-4^{\prime \prime}\right), 5.21$ (dd, $1 \mathrm{H}, J=7.7,10.5 \mathrm{~Hz}$, H-2"), 5.15 (dd, 1H, $J=8.0,10.3$ Hz, H-2'), 5.09 (dd, 1H, J=10.3, 3.4 Hz, H-3'), 5.04, 4.60 (2d, $2 \mathrm{H}, J=11.6 \mathrm{~Hz}, \mathrm{PhCH}_{2}$ ), 4.93 (dd, 1H, $\left.J=10.5,3.4 \mathrm{~Hz}, \mathrm{H}-3^{\prime \prime}\right), 4.92$ (d, 1H, J= 7.7 Hz, H-1"), $4.85\left(\mathrm{~d}, 1 \mathrm{H}, J=8.0 \mathrm{~Hz}, \mathrm{H}^{-1}{ }^{\prime}\right), 4.56$ (d, 1H, $\left.J=7.7 \mathrm{~Hz}, \mathrm{H}-1\right), 4.35$ (dd, 1H, $J=1.6,12.3 \mathrm{~Hz}, \mathrm{H}-$ 6a), 4.26 (dd, 1H, $J=3.7,0.9 \mathrm{~Hz}, \mathrm{H}-4), 4.19$ (dd, 1H, $J=7.7,9.7 \mathrm{~Hz}, \mathrm{H}-2), 4.18$ (dd, 1H, $J=$ 6.7, $\left.11.2 \mathrm{~Hz}, \mathrm{H}-6^{\prime} \mathrm{a}\right), 4.14$ (dd, 1H, $J=6.4,11.2 \mathrm{~Hz}, \mathrm{H}-66^{\prime} \mathrm{b}$ ), 4.09 (dd, 1H, J = 7.5, $11.2 \mathrm{~Hz}$, H6"a), 4.06 (dd, 1H, $J=1.7,12.3 \mathrm{~Hz}, \mathrm{H}-6 \mathrm{~b}), 3.97$ (dd, H, J = 6.2, $11.2 \mathrm{~Hz}, \mathrm{H}-66^{\prime \prime}$ ), 3.91 (m, 2H, H-5', H-3), 3.63 (ddd, 1H, $J=1.1,6.4,7.4$ Hz, H-5"), 3.40 (m, 1H, H-5), 2.16, 2.14, 2.04, 2.03, 1.97, 1.96 (6s, 24H, $\left.\mathrm{CH}_{3} \mathrm{CO}\right) ;{ }^{13} \mathrm{C} \mathrm{NMR}\left(\mathrm{CDCl}_{3}, 50.3 \mathrm{MHz}\right): \delta 170.2,170.1,170.0,169.6,169.3$ (CO), 137.7, 137.5, 128.7, 128.3, 127.9, 127.6, 127.3, 126.1 (arom.), $101.0(\mathrm{PhCH}), 100.7,100.3$ (C-1', C-1"), 99.7 (C-1), 77.6, 76.3 (C-2, C-3), 75.6, 71.0, 70.9, 70.8, 70.6, 70.4 ( $\left.\mathrm{PhCH}_{2}\right), 70.2$, 69.6, 68.9 (C-6), 67.2, 66.9, 66.3; 61.3, 60.9 (C-6', C-6"); 20.9, 20.6, $20.5\left(\mathrm{CH}_{3} \mathrm{CO}\right)$.

Anal. Calcd. $\mathrm{C}_{48} \mathrm{H}_{58} \mathrm{O}_{24}$ : C, 56.58; H, 5.74. Found: C, 56.40; H, 5.78.

Benzyl 2,3-di-O-(2,3,4,6-tetra-O-acetyl- $\beta$-D-galactopyranosyl)- $\beta$-D-galactopyranoside (11).To a solution of benzyl 4,6-O-benzylidene-2,3- di - O-2,3,4,6 - tetra- $O$-acetyl- $\beta$-D-galactopyranosyl)- $\beta$-Dgalactopyranoside $(\mathbf{1 0}, 0.71 \mathrm{~g}, 0.69 \mathrm{mmol})$ in acetic acid $(5 \mathrm{~mL})$ warmed at $80{ }^{\circ} \mathrm{C}, \mathrm{H}_{2} \mathrm{O}(2.5 \mathrm{~mL})$ was slowly added with stirring until turbidity. After $1 \mathrm{~h}$, the mixture was cooled and extracted with $\mathrm{CH}_{2} \mathrm{Cl}_{2}(3 \times 100 \mathrm{ml})$. The organic phase was washed with saturated aqueous $\mathrm{NaHCO}_{3}(2 \mathrm{x}$ $80 \mathrm{ml})$, water $(2 \times 100 \mathrm{ml})$, dried $\left(\mathrm{MgSO}_{4}\right)$ and evaporated. The crude product was purified by recrystallization from a mixture of hexane-EtOAc-toluene (80:15:5) to give $0.49 \mathrm{~g}$ of 11 (75\%): $\operatorname{mp} 112-115^{\circ} \mathrm{C},[\alpha]_{\mathrm{D}}-25.2^{\circ}\left(\mathrm{c} 1, \mathrm{CHCl}_{3}\right) .{ }^{1} \mathrm{H}$ NMR $\left(\mathrm{CDCl}_{3}, 500 \mathrm{MHz}\right): \delta$ 7.4-7.29 (m, $\left.5 \mathrm{H}\right), 5.38$ $\left(\mathrm{d}, 1 \mathrm{H}, J=3.4 \mathrm{~Hz}, \mathrm{H}-4^{\prime}\right), 5.31$ (d, $\left.1 \mathrm{H}, J=3.5 \mathrm{~Hz}, \mathrm{H}-4^{\prime \prime}\right), 5.20$ (dd, $1 \mathrm{H}, J=7.8,10.3 \mathrm{~Hz}, \mathrm{H}-2^{\prime \prime}$ ), $5.16\left(\mathrm{dd}, 1 \mathrm{H}, J=8.0,10.5 \mathrm{~Hz}, \mathrm{H}-2^{\prime}\right), 5.11\left(\mathrm{dd}, 1 \mathrm{H}, J=10.5,3.4 \mathrm{~Hz}, \mathrm{H}-3^{\prime}\right), 4.99,4.65$ (2d, 2H, $J$ $\left.=11.9 \mathrm{~Hz}, \mathrm{PhCH}_{2}\right), 4.98$ (dd, 1H, J=10.3, 3.5 Hz, H-3"), 4.87 (d, 1H, J=8.0 Hz, H-1'), 4.81 (d, $\left.1 \mathrm{H}, J=7.8 \mathrm{~Hz}, \mathrm{H}-1^{\prime \prime}\right), 4.52$ (d, 1H, $\left.J=7.8 \mathrm{~Hz}, \mathrm{H}-1\right), 4.16-3.94$ (m, 9H), 3.82 (dd, 1H, $J=9.1$, 
$3.5 \mathrm{~Hz}, \mathrm{H}-3), 3.80$ (m. 1H), 3.67, 3.51 (2m, 2H), 2.84 (bs, 1H, OH), 2.18, 2.14, 2.11, 2.07, 2.06, 2.00, 1.99, $1.97\left(8 \mathrm{~s}, 24 \mathrm{H}, \mathrm{CH}_{3} \mathrm{CO}\right) ;{ }^{13} \mathrm{C} \mathrm{NMR}\left(\mathrm{CDCl}_{3}, 125 \mathrm{MHz}\right): \delta 170.4,170.2,170.1,170.0$, 169.9, 169.8, 169.4 ( $\left.\mathrm{CH}_{3} \mathrm{CO}\right), 137.0,128.3,127.5,127.4$ (arom.), 100.9, 100.3, 99.4 (C-1, C-1', C-1"), 81.1 (C-2), 76.3 (C-3), 73.7, 71.2, 70.9, $70.8\left(\mathrm{PhCH}_{2}\right), 70.6,70.5,70.1,69.6,68.5,67.1$ (x2); 62.3, 61.5, 61.0 (C-6, C-6', C-6"); 21.0, 20.6, $20.5\left(\mathrm{CH}_{3} \mathrm{CO}\right)$.

Benzyl 4,6-di- $O$-acetyl-2,3-di- $O$-(2,3,4,6-tetra- $O$-acetyl- $\beta$-D-galactopyranosyl)- $\beta$-D-galactopyranoside (12). To a stirred solution of benzyl 2,3-di-O-(2,3,4,6-tetra-O-acetyl- $\beta$-Dgalactopyranosyl)- $\beta$-D-galactopyranoside $(11,0.73 \mathrm{~g}, 0.785 \mathrm{mmol})$ in dry pyridine $(3.7 \mathrm{~mL})$ cooled to $0{ }^{\circ} \mathrm{C}$, acetic anhydride was slowly added $(3.7 \mathrm{ml})$ and the solution was stirred at $5{ }^{\circ} \mathrm{C}$ for $16 \mathrm{hs}$. The mixture was cooled to $0^{\circ} \mathrm{C}, \mathrm{MeOH}(5 \mathrm{ml})$ was added and the stirring continued for $30 \mathrm{~min}$. The solution was diluted with $\mathrm{CH}_{2} \mathrm{Cl}_{2}(200 \mathrm{ml})$ and washed with $10 \% \mathrm{HCl}$, water, saturated aqueous $\mathrm{NaHCO}_{3}$ and water. The organic phase was dried $\left(\mathrm{MgSO}_{4}\right)$, filtered and concentrated. Purification by chromatography on a silica gel short column (hexane-EtOAc 2:3) gave $0.75 \mathrm{~g}$ of $\mathbf{1 2}$ as a foamy solid (90\% yield) that crystallized from 5:1 hexane-ether. R $f 0.43$ (1:1 toluene-EtOAc), mp 94-97 ${ }^{\circ} \mathrm{C},[\alpha]_{\mathrm{D}}-15.3^{\circ}$ (c 1, $\left.\mathrm{CHCl}_{3}\right) ;{ }^{1} \mathrm{H} \mathrm{NMR}\left(\mathrm{CDCl}_{3}, 500 \mathrm{MHz}\right): \delta$ 7.41-7.30 (m, $5 \mathrm{H}), 5.38$ (m, 1H, H-4), 5.34 (dd, 1H, $\left.J=3.1,1.3 \mathrm{~Hz}, \mathrm{H}-4^{\prime}\right), 5.31$ (dd, 1H, $J=3.5$, $\left.1.3 \mathrm{~Hz}, \mathrm{H}-4^{\prime \prime}\right), 5.17$ (dd, 1H, $\left.J=7.8,10.5 \mathrm{~Hz}, \mathrm{H}-2^{\prime \prime}\right), 5.10$ (dd, $\left.1 \mathrm{H}, J=7.0,10.4 \mathrm{~Hz}, \mathrm{H}-2^{\prime}\right), 5.09$ (dd, $\left.1 \mathrm{H}, J=10.4,3.1 \mathrm{~Hz}, \mathrm{H}-3^{\prime}\right), 5.00,4.62$ (2d, 2H, $J=11.8 \mathrm{~Hz}, \mathrm{PhCH}_{2}$ ), 4.92 (dd, 1H, $J=10.5$, $\left.3.5 \mathrm{~Hz}, \mathrm{H}-3^{\prime \prime}\right), 4.84$ (d, 1H, J=7.8 Hz, H-1"'), 4.76 (d, 1H, J=7.0 Hz, H-1'), 4.55 (d, 1H, J = 7.5 Hz, H-1), 4.16 (dd, 1H, J = 5.7, 11.7 Hz, H-6a), 4.14 (d, 2H, J = 6.7 Hz, H-6'a, H-6'b), 4.10 (dd, $\left.1 \mathrm{H}, J=7.7,11.2 \mathrm{~Hz}, \mathrm{H}-6^{\prime \prime} \mathrm{a}\right), 4.09$ (dd, 1H, $\left.J=7.0,11.7 \mathrm{~Hz}, \mathrm{H}-6 \mathrm{~b}\right), 4.01$ (dd, H, $J=6.0,11.2$ Hz, H-6'b), 3.91 (m, 2H, H-2, H-3), 3.89 (dt, 1H, J = 1.3, 6.7 Hz, H-5'), 3.76 (ddd, 1H, $J=1.1$, 5.7, $7.0 \mathrm{~Hz}, \mathrm{H}-5), 3.62$ (m, 1H, $\left.J=1.3,6.0,7.7 \mathrm{~Hz}, \mathrm{H}-5^{\prime \prime}\right), 2.17,2.15,2.10,2.08,2.07,2.05$, 2.00, 1.97 (8s, 30H, $\left.\mathrm{CH}_{3} \mathrm{CO}\right) ;{ }^{13} \mathrm{C} \mathrm{NMR}\left(\mathrm{CDCl}_{3}, 125 \mathrm{MHz}\right): \delta 170.5,170.3,170.3,170.1,170.1$, 170.0, 169.9, 169.8, 169.4, $169.1\left(\mathrm{CH}_{3} \mathrm{CO}\right), 137.0,128.4,127.9,127.4$, (arom.), $100.8(\mathrm{C}-1)$, $99.9\left(\mathrm{C}-1^{\prime}\right), 99.7\left(\mathrm{C}-1^{\prime \prime}\right), 77.8$ (C-2), $76.3(\mathrm{C}-3), 71.1,71.0\left(\mathrm{C}-3^{\prime}, \mathrm{C}-5^{\prime \prime}\right), 70.9\left(\mathrm{PhCH}_{2}\right), 70.8(\mathrm{C}-$ 5'), 70.6 (C-3', C-5"'), 69.9 (C-2"), 69.7 (C-2'), 68.8 (C-4), 67.0 (C-4'), 66.9 (C-4"), 62.2 (C-6); 61.0, 60.9 (C-6', C-6" $)$; 20.9, 20.7, 20.6, $20.5\left(\mathrm{CH}_{3} \mathrm{CO}\right)$. The assignments were supported by homo and heteronuclear correlation spectroscopy experiments.

Anal. Calcd for $\mathrm{C}_{45} \mathrm{H}_{58} \mathrm{O}_{26}$ : C, 53.25; H, 5.76. Found: C, 53.25; H, 5.82.

Benzyl 2,3-di-O- $\beta$-D-galactopyranosyl- $\beta$-D-galactopyranoside (13). To a flask containing benzyl 4,6-di- $O$-acetyl-2,3-di- $O$-(2,3,4,6-tetra- $O$-acetyl- $\beta$-D-galactopyranosyl)- $\beta$-D-galactopyranoside (12, $295 \mathrm{mg}, 0.291 \mathrm{mmol})$ was added $3 \mathrm{ml}$ of a cooled $0.4 \mathrm{M}$ sodium methoxide solution. After $45 \mathrm{~min}$ of stirring at $0{ }^{\circ} \mathrm{C}$, a precipitate was formed and TLC examination showed only a more polar compound than 12. Water was added and the resulting solution was decationized by elution through a column of Amberlite IR $120 \mathrm{H}^{+}$resin. Evaporation of the solution gave $0.171 \mathrm{~g}$ of a white crystalline solid (99\% yield) which was recrystallized from methanol-water: $\mathrm{R} f 0.35$ (7:1:1 n-propanol-ethanol-water), mp 286-290 ${ }^{\circ} \mathrm{C},[\alpha]_{\mathrm{D}}-0.5^{\circ}$ (c 1 , water); ${ }^{1} \mathrm{H}$ NMR $\left(\mathrm{D}_{2} \mathrm{O}, 500 \mathrm{MHz}\right): \delta 7.46-7.33(\mathrm{~m}, 5 \mathrm{H}$, arom.), 4.93, $4.72(2 \mathrm{~d}, 2 \mathrm{H}, J=11.7 \mathrm{~Hz}$, $\left.\mathrm{PhCH}_{2}\right) ; 4.71$, (d, 1H, J = 8.0 Hz, H-1"); 4.59 (d, 2H, J= 7.3, Hz, H-1, H-1'); 4.15 (d, 1H, J = 2.4 
Hz, H-4); 3.90 (m, 2H, H-2, H-3); 3.86 (d, 1H, J = 3.3 Hz, H-4' or 4"); 3.84 (d, 1H, J=3.3 Hz, H-4' or H-4"); 3.76-3.64 (m, 5H); 3.63-3.49 (m, 7H); 3.46 (dd, 1H, J=8.0, $10.0 \mathrm{~Hz}, \mathrm{H}-2^{\prime}$ or H$\left.2^{\prime \prime}\right) ;{ }^{13} \mathrm{C}$ NMR (D $\left.2 \mathrm{O}, 125 \mathrm{MHz}\right): \delta 137.4,129.2,128.9$ (arom.), 104.3, 103.7 (C-1', C-1"), 101.3 (C-1), 82.9 (C-2), 77.5 (C-3), 75.7, 75.6, 75.2, 73.4, 73.3 (C-3', C-3", C-5, C-5', C-5"), 72.2, 71.7 $\left(\mathrm{C}-2^{\prime}, \mathrm{C}-2^{\prime \prime}\right), 71.8\left(\mathrm{PhCH}_{2}\right)$; 69.3 (C-4); 69.2, 69.1 (C-4', C-4"); 61.5 61.4, 61.2 (C-6, C-6', C-6"). The assignments were supported by heteronuclear correlation spectroscopy experiments. Anal. Calcd for $\mathrm{C}_{25} \mathrm{H}_{38} \mathrm{O}_{16} .1 \frac{1}{2} \mathrm{H}_{2} \mathrm{O}$ : C, 48.31; H, 6.65. Found: $\mathrm{C}, 48.25 ; \mathrm{H}, 6.71$.

2,3-Di-O- $\beta$-D-galactopyranosyl- $\beta$-D-galactose (14). To a solution of benzyl 2,3-di- $O$ - $\beta$-Dgalactopyranosyl- $\beta$-D-galactopyranoside $(13,92.6 \mathrm{mg}, 0.155 \mathrm{mmol})$ in water $(4 \mathrm{~mL})$, was added $10 \% \mathrm{Pd} / \mathrm{C}$ Deguzza type E101 NE/W (80 mg), and the suspension was hydrogenated for $3 \mathrm{~h}$ at $1.5 \mathrm{~atm}$. The catalyst was filtered and the solution was passed through a C-8 reverse phase cartridge and eluted with water. The solution was concentrated at r.t. to give $75.4 \mathrm{mg}$ of a glassy solid (14, $96 \%)$ that crystallized upon addition of methanol: $\mathrm{R} f$ 0.3 (7:1:2 n-propanol-ethanolwater), mp $175-180^{\circ} \mathrm{C},[\alpha]_{\mathrm{D}}+33.2^{\circ}$ ( $c$ 1, water). Analysis of the spectra showed that 14 was a mixture of the four configurations at the reducing end: ${ }^{1} \mathrm{H} \mathrm{NMR}\left(\mathrm{D}_{2} \mathrm{O}, 500 \mathrm{MHz}\right): \delta$ anomeric region $5.41(\mathrm{~d}, 0.12 \mathrm{H}, J=1.0 \mathrm{~Hz}, \mathrm{H}-1 \beta$ furanosic $) ; 5.38,(\mathrm{~d}, 0.58 \mathrm{H}, J=3.8 \mathrm{~Hz}, \mathrm{H}-1 \alpha$ pyranosic); $5.33(\mathrm{~d}, 0.05 \mathrm{H}, J=4.5 \mathrm{~Hz}, \mathrm{H}-1 \alpha$ furanosic); $4.73(\mathrm{~d}, 0.25 \mathrm{H}, J=8.00 \mathrm{~Hz}, \mathrm{H}-1 \beta$ pyranosic); 4.63 (d, $0.58 \mathrm{H}, J=7.8 \mathrm{~Hz}, \mathrm{H}-1^{\prime}$ or H-1"); 4.62 (d, 0.25H, $J=7.8 \mathrm{~Hz}, \mathrm{H}-1^{\prime}$ or H-1"), 4.59 (d, 0.25H, $J$ $=7.6 \mathrm{~Hz}, \mathrm{H}-1^{\prime}$ or H-1"), 4.57 (d, 0.58H, $J=7.8 \mathrm{~Hz}, \mathrm{H}-1^{\prime}$ or H-1"); ${ }^{13} \mathrm{C}$ NMR $\left(\mathrm{D}_{2} \mathrm{O}, 125 \mathrm{MHz}\right): \delta$ anomeric region 104.7, 104.6, 103.5, 103.4, 102.9, 102.6 (C-1', C-1"), 101.1 (C-1 $\beta$ furanosic), 95.7 (C-1 $\beta$ pyranosic), 95.6 (C-1 $\alpha$ furanosic), 92.6 (C-1 $\alpha$ pyranosic).

Anal. Calcd for $\mathrm{C}_{18} \mathrm{H}_{32} \mathrm{O}_{16} \cdot \mathrm{H}_{2} \mathrm{O}$ : C, 41.38; H, 6.56. Found: $\mathrm{C}, 41.36 ; \mathrm{H}, 6.50$.

\section{Acknowledgements}

This work was supported by grants from Agencia Nacional de Promoción Científica y Tecnológica (ANPCyT, BID 1201/OC-AR, PICT 06-05133), Universidad de Buenos Aires and Fundación Antorchas. C. Gallo-Rodriguez and R. M. de Lederkremer are research members of CONICET. V. M. Mendoza is research fellow from ANPCyT.

\section{References}

1. Acosta-Serrano, A.; Almeida, I. C.; Freitas-Junior, L. H.; Yoshida, N.; Schenkman, S. Mol. Biochem. Parasitol. 2001, 114, 143 and references there in.

2. Previato, J. O.; Jones, C.; Gonçalves, L. P.; Wait, R.; Travassos, L. R.; Mendonça-Previato, L. Biochem. J. 1994, 301, 151.

3. Acosta-Serrano, A.; Schenkman, S.; Yoshida, N.; Mehlert, A.; Richardson, J. M.; Ferguson, M. A. J. Biol. Chem. 1995, 270 , 27244. 
4. Schenkman, S.; Eichinger, D.; Pereira, M. E.; Nussenzweig, V. Annu. Rev. Microbiol. 1994, 48, 499.

5. Parodi, A. J.; Pollevick, G. D.; Mautner, M.; Buschiazzo, A.; Sanchez, D. O.; Frasch, A. C. EMBO J. 1992, 11, 1705.

6. Gallo-Rodriguez, C.; Varela, O.; Lederkremer, R. M. J. Org. Chem. 1996, 61, 1886.

7. Gallo-Rodriguez, C.; Varela, O.; Lederkremer, R. M. Carbohydr. Res. 1998, 305, 163.

8. Gallo-Rodriguez, C.; Gil Libarona, M. A.; Mendoza, V. M.; Lederkremer, R. M. Tetrahedron 2002, 58, 9373.

9. Agrellos, O. A.; Jones, C.; Todeschini, A. R.; Previato, J. O., Mendonca-Previato, L. Mol. Biochem. Parasitol. 2003, 126, 93.

10. D’Auria, M. V.; Gomez Paloma, L.; Minale, L.; Riccio, R. Tetrahedron 1992, 48, 491.

11. Collins, P. M.; Eder, H. J. Chem. Soc. Perkin Trans. I 1983, 927

12. Fleet, G. W. J.; Son, J. C. Tetrahedron 1988, 44, 2637.

13. (a) Schmidt, R. R.; Kinzy, W. Adv. Carbohydr. Chem. Biochem. 1994, 50, 21. (b)Schmidt, R. R. In Modern Methods in Carbohydrate Synthesis; Khan, S. H., O’Neil, P. A., Eds.; Harwood Academic Publishers: Amsterdam, 1996; pp 20-54. (c) Schmidt, R. R., Jung, K.-H. In Preparative Carbohydrate Chemistry; Hanessian, S., Ed.; Marcel Dekker, 1997; pp 283-312.

14. (a) Schmidt, R. R.; Stumpp, M. Liebigs Ann. Chem. 1983, 1249. (b) Amwam-Zollo, P. H.; Sinaÿ, P. Carbohydr. Res. 1986, 150, 199.

15. Lerner, L. M. Methods Carbohydr. Chem. 1972, 6, 131.

16. Gandolfi-Donadio, L.; Gallo-Rodriguez, C.; Lederkremer, R. M. J. Org. Chem. 2002, 67, 4430.

17. Zhang, J.; Kováč, P. J. Carbohydr. Chem. 1999, 18, 461.

18. Kobayashi, Y.; Shiozaki, M.; Ando, O. J. Org. Chem. 1995, 60, 2570.

19. Takeo, K.; Kitajima, M.; Fukatsu, T. Carbohydr. Res. 1983, 112, 158. 